\title{
25-gauge histology needle versus 22-gauge cytology needle in endoscopic ultrasonography-guided sam- pling of pancreatic lesions and lymphadenopathy
}

Authors

Institutions
Georgios Mavrogenis ${ }^{1,2}$, Birgit Weynand ${ }^{3}$, Alain Sibille ${ }^{1}$, Hocine Hassaini ${ }^{1}$, Pierre Deprez ${ }^{2}$, Cédric Gillain ${ }^{1}$, Philippe Warzée $^{1}$

Institutions are listed at the end of article. submitted 13. June 2014 accepted after revision 26. August 2014

\section{Bibliography}

Dol http://dx.doi.org/ 10.1055/s-0034-1390889

Published online: 12.12 .2014 Endoscopy International Open 2015; 03: E63-E68

(c) Georg Thieme Verlag KG Stuttgart · New York E-ISSN 2196-9736

\section{Corresponding author} Georgios Mavrogenis, MD Grand Hôpital de Charleroi Site Notre Dame

3 Grand Rue 6000 Charleroi Belgium

Fax: +3271102779

mavrogenis@gmail.com
Background and study aims: A new 25-gauge (G) endoscopic ultrasound-guided fine-needle aspiration (EUS-FNA) device (EchoTip ProCore; Cook Medical, Bloomington, Indiana, USA) has been developed, which features a hollowed-out reverse bevel to trap core samples. However, data on the differences between the diagnostic yield of the 25G EchoTip ProCore and that of a 22G standard needle are limited.

Patients and methods: This pilot study included 27 patients referred during an 11-month period for EUS-FNA of pancreatic masses and enlarged lymph nodes adjacent to the upper gastrointestinal tract. Each lesion was punctured once by both a 25G EchoTip ProCore needle and a 22G standard needle (EchoTip; Cook Medical) with capillary sampling. Blinded histocytologic analyses were conducted. The final diagnosis was based on FNA findings of malignant cells, pathologic analysis of the surgical specimen, and/or radiologic and clinical follow-up of at least 7 months.

\section{Introduction \\ $\nabla$}

Endoscopic ultrasound-guided fine-needle aspiration (EUS-FNA) has become a mainstay diagnostic technique for the evaluation of lesions arising from the pancreas and upper gastrointestinal tract as well as adjacent structures, including lymph nodes and the liver. EUS-FNA is safe and has a diagnostic accuracy of $60 \%$ to $90 \%$ [1-3]. The diagnostic yield of EUS-FNA is influenced by several factors: the nature of the target lesion, experience of the endoscopist, presence of an onsite cytopathologist, and various technical aspects, such as needle size and number of passes. In order to limit the influencing parameters, a novel needle design was introduced in 2010-2011 that features a hollowed-out reverse bevel to trap core samples (EchoTip ProCore; Cook Medical, Bloomington, Indiana, USA). A potential advantage of this type of needle is the acquisition of lar-
Results: A total of 28 EUS-FNA procedures targeting masses of the pancreas $(n=19)$ and lymph nodes $(n=9)$ were performed. No complications were encountered. Single-pass sensitivity rates for pancreatic and lymph node malignancy were equal for the needle types: $89.5 \%$ (95\%CI $66.82-$ 98.39) and 66\% (95\%CI 24.1-94), respectively. There were no significant differences between the needles in terms of EUS visualization $(P=0.125)$, amount of blood contamination $(P=0.705)$, macroscopic quantity of the material $(P=0.858)$, quality of the cytology $(P=0.438)$, and adequacy and accuracy of the cell block material $(P=0.220)$.

Conclusions: Both needles were safe and successful in terms of a high diagnostic yield, with similar histocytologic results.

The results of this study were presented at Digestive Disease Week (DDW) 2014, Chicago, Illinois. This trial was registered at ClinicalTrials.gov (B027201316271).

ger amounts of tissue with preserved architecture. Preliminary results on the performance of the 19-gauge (G), 22G, and 25G ProCore needles were promising, with reported high diagnostic accuracy rates of $89.6 \%$ [4], 88.5\% [5], and 86\% [6], respectively. However, comparative studies of the $22 \mathrm{G}$ ProCore needle versus the $22 \mathrm{G}$ standard cytology needle showed similar results for the cytology parameters, amount of blood contamination, and diagnostic adequacy and accuracy of the cell block material [7-9]. Fewer data are available on the diagnostic yield of the 25G ProCore needle. Preliminary data demonstrated an $83 \%$ first-pass sensitivity for pancreatic malignancy, with a cumulative sensitivity of $96 \%$ after three passes [6]. Histologic diagnosis was possible in $63 \%$ after a single pass and in $80 \%$ after multiple passes [6]. Taking into account the results of a recent meta-analysis [10] that shows a potential advantage of the $25 \mathrm{G}$ needle over the $22 \mathrm{G}$ needle 

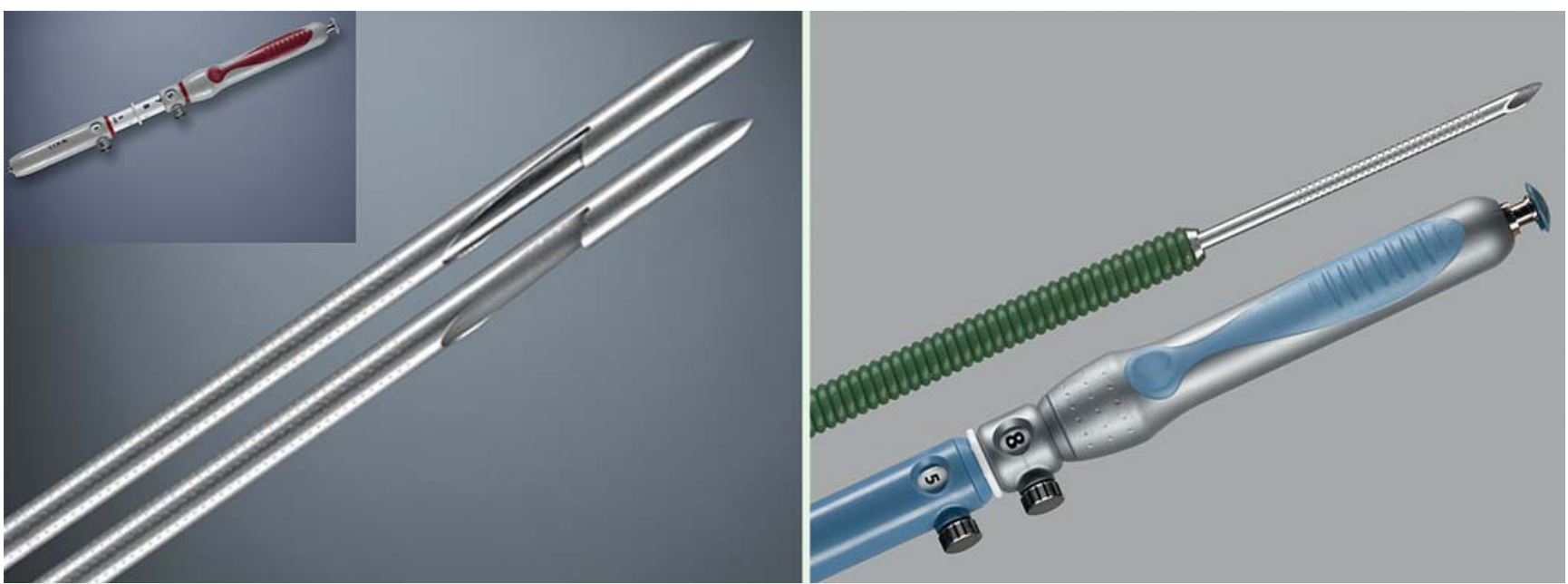

Fig. 1 The 25G EchoTip ProCore histology needle (left), which has a hollowed-out reverse bevel, and the 22G EchoTip standard cytology needle (right). Photos supplied by Cook Medical.

for the diagnosis of pancreatic malignancy, the idea of a 25G needle that can obtain histologic specimens sounds attractive. Therefore, we designed a prospective study comparing the most commonly used 22G standard cytology needle versus the 25G EchoTip ProCore needle in the same group of lesions in terms of differences in accuracy, technical performance, and quality and quantity of the cytology and cell block specimen obtained.

\section{Materials and methods}

$\nabla$

This pilot study included patients with pancreatic mass lesions or lymphadenopathy who were referred for EUS-FNA sampling at our hospital (Grand Hôpital de Charleroi, Charleroi, Belgium) between December 2012 and October 2013.Exclusion criteria included the following: cystic lesion, coagulation disorder (international normalized ratio $>1.5$, platelet count $<50000 / \mathrm{mm}^{3}$ ), pregnancy, age $<18$ years, and refusal or inability to provide informed consent. Patients were monitored closely for possible complications after the procedure. The study was approved by the Grand Hôpital de Charleroi review board, and written informed consent was obtained from all patients for participation in the study. This trial was registered at ClinicalTrials.gov (B027201316271).

A linear array echoendoscope (UCT160; Olympus Medical Systems, Tokyo, Japan) connected to a processor featuring color Doppler function (ProSound Alpha 7; Hitachi Medical Systems Europe [Aloka], Zug, Switzerland) was used for all procedures, which were carried out by four experienced endosonographers (A.S., P.W., H.H., and C.G.) and one gastroenterology fellow (G.M.) while the patients were under propofol sedation. The gastroenterology fellow was present during all procedures in order to ensure adherence to the study protocol. After the lesion had been carefully inspected and vessel interposition along the puncture route excluded by color Doppler, EUS-FNA was performed with both a 22G (EchoTip) needle and a 25G EchoTip ProCore needle ( $\bullet$ Fig. 1). The order in which the needles were used was according to computer-generated randomization. During each puncture, the needle went through the lesion, and 15 to 20 to-andfro movements were made with minimal negative pressure simultaneously applied by pulling the needle stylet slowly and continuously (slow-pull technique) [6]. After the second puncture, if the acquired specimen was not adequate, EUS-FNA was repeated with one of the two needles until an adequate specimen had been obtained. No on-site cytopathologic evaluation was done. FNA material was recovered in a vial containing $15 \mathrm{~mL}$ of ThinPrep PreservCyt Solution (Hologic, Marlborough, Massachusetts, USA) by flushing with $5 \mathrm{~mL}$ of this solution and, if needed, by pushing the stylet through the needle. The specimens obtained were labeled according to the aspiration sequence and sent to a single experienced cytopathologist (B.W.), who was blinded to the types of needles used. All samples were processed in the same way. One monolayer was obtained with ThinPrep 2000 and stained with Papanicolaou stain (cytologic specimen); the leftover material was centrifuged and the clot retrieved in agar, fixed in formalin for at least 2 hours, and embedded in paraffin to obtain a cell block (histologic specimen). One 5 - $\mu$ m-thick slide stained with hematoxylin and eosin was available for every cell block. Immunocytochemistry was applied on the cell block if needed for the diagnosis. Only the results of the first and second punctures were compared.

The quality of the obtained cytologic and histologic specimens was reported by the cytopathologist according to the following grading system: insufficient material or contaminated material (score of 0 ), rare diagnostic cells (score of 1 ), diagnostic cells at every field with 10 times magnification (score of 2), or diagnostic cells at every field with 20 times magnification (score of 3 ). The primary outcome parameter was the percentage of cases in which the pathologist classified the quality of the sample as sufficient for histocytologic evaluation (score $\geq 1$ ). This grading system, although not published as such, is very easy to apply to a cytologic slide, especially when the specimen has been obtained with a monolayer technique. It is also easily reproducible because one needs only to record the magnification at which diagnostic cells are seen filling the microscopic field.

Secondary outcomes included comparisons of several performance parameters. The visibility of each needle was qualitatively scored as optimal or suboptimal and the ease of puncture as easy or difficult $[6,11]$. The amount of blood contamination was scored qualitatively as no blood (score of 1 ), small quantity of blood (score of 2), or large quantity of blood (score of 3) [12]. The quantity of the specimens was graded as small (score of 1) 
in the presence of fragments with no visible core, medium when one or two cores were visible (score of 2), or excellent when at least three cores were visible (score of 3 ).

A final diagnosis of malignancy or benignancy was made according to one of the following reference methods: (1) definite benign or malignant histologic diagnosis based on surgical resection specimens from patients who had undergone surgery, (2) cytology or histology findings with definite proof of malignancy in patients with unresectable tumors according to imaging findings and compatible clinical follow-up, and (3) cytology or histology findings without proof of malignancy and a minimum clinical and radiologic follow-up of 7 months.

For comparison of continuous data, a paired $t$ test was performed if a normal distribution was shown, and the Wilcoxon rank sum test was carried out if normality could not be demonstrated. McNemar's test was used for dichotomous categorical data. For all tests, a $P$ value of less than 0.05 was regarded as statistically significant with SPSS 17.0 for Windows (SPSS Inc., Chicago, Illinois, USA).

\section{Results}

\section{$\nabla$}

A total of 28 EUS-FNA procedures targeting lesions of the pancreas $(n=19)$ and lymph nodes $(n=9)$ were performed in 27 patients ( 18 women, 9 men) with a median age of 69 years (range, $38-88$ ). The final diagnoses were pancreatic adenocarcinoma $(n=18)$, pancreatic neuroendocrine tumor $(n=1)$, malignant lymphadenopathy $(n=6)$, and benign lymphadenopathy $(n=3)$. No benign pancreatic lesions were encountered.

In the subgroup of patients with pancreatic lesions, the median tumor size was $39 \mathrm{~mm}$ (range, 10-70). Of these pancreatic lesions, 10 were punctured through the duodenum and 9 were punctured through the stomach.

In the subgroup of patients with lymphadenopathy, the median lymph node size was $24 \mathrm{~mm}$ (range, 15-45). Of these lesions, 6 were punctured through the esophagus and 3 through the duodenum.

Final diagnoses were made on the basis of surgery in 3 cases, positive FNA for malignancy with a compatible clinical course in 23 cases, and negative FNA for malignancy with at least 7 months of follow-up in 2 cases. No procedure-related complications were seen.

In terms of EUS visualization, visualization was suboptimal in 16 $\%$ of punctures with the $25 \mathrm{G}$ needle versus $0 \%$ of punctures with the $22 \mathrm{G}$ needle; however, this difference was not statistically significant $(P=0.125)(\diamond$ Videos 1,2$)$. No relevant differences were found regarding the ease of puncture $(P=0.688)$, amount of blood contamination $(P=0.705)$, macroscopic quantity of the material $(P=0.858)$, and quality of the cytologic $(P=0.438)$ and histologic $(P=0.220)$ specimens ( $\bullet$ Table 1$)$. Subgroup characteristics regarding the adequacy of histocytologic material for the two needle types and different types of lesions are shown in $\bullet$ Table 2 .

\section{Video 1}

Endoscopic ultrasound-guided fine-needle aspiration of a pancreatic mass with a 25G EchoTip ProCore needle.

online content including video sequences viewable at:

www.thieme-connect.de
In the subgroup of patients with pancreatic cancer, each needle missed two cases. The first case was a patient with adenocarcinoma of the head of the pancreas. Transduodenal puncture with the $25 \mathrm{G}$ needle showed rare benign cells (cytology score $=1$, cell block score $=1$ ), while puncture with the $22 \mathrm{G}$ needle was noncontributive (cytology score $=0$, cell block score $=0$ ). Because of the strong suspicion of malignancy, a follow-up EUS-FNA a few weeks later was performed with a standard $22 \mathrm{G}$ needle and confirmed the diagnosis of pancreatic adenocarcinoma. Each needle missed one other case of pancreatic adenocarcinoma of the head of the pancreas punctured through the duodenum because of insufficient histocytologic material (total histocytologic score $=0$ ). Therefore, the single-pass sensitivity for pancreatic neoplasia for both needles was $89.5 \%$ ( $95 \%$ CI $66.82-98.39$ ).

In the subgroup of patients with lymphadenopathy, a total of two false-negative results for malignancy were obtained with both needles that concerned the same patients. The first patient had gallbladder cholangiocarcinoma and perihepatic lymph nodes suspicious for malignancy that were $2 \mathrm{~cm}$ in size. Transduodenal puncture with both needles resulted into noncontributive histocytologic material (total histocytologic score $=0$ ). Follow-up imaging was compatible with metastatic lymph nodes. The second patient had lung cancer and mediastinal lymph nodes suspicious for malignancy ( $4 \mathrm{~cm}$ in size). EUS-FNA with both needles showed rare benign cells (cytology score $=1$, histology score $=1$ ). However, clinical and radiologic follow-up was compatible with metastatic lymph nodes. Therefore, the single-pass sensitivity, specificity, positive predictive value, negative predictive value, and accuracy for malignancy were equal for the needle types: $66 \%(95 \%$ Cl $24.1-94), 100 \%$ (95\%CI $30.9-100), 44.4 \%$ (95\%CI $39.5-100$ ), $60 \%$ (95\%CI $17-92.7$ ), and $84.8 \%$ (95\%CI $67.3-94.2$ ), respectively.

\section{Discussion}

\section{$\nabla$}

Overall, EUS-FNA is highly effective for most pancreatic tumors and solid malignancies adjacent to the upper gastrointestinal tract, with reliable sensitivity, specificity, and overall diagnostic accuracy of $60 \%$ to $90 \%$ [1 - 3]. However, FNA cytology specimens may not be adequate in cases in which the diagnosis relies on tissue architecture, such as autoimmune pancreatitis, lymphomas, gastrointestinal stromal tumors, and well-differentiated adenocarcinomas $[1,13]$.

In order to procure larger amounts of tissue with preserved architecture that would enable histologic analysis, a novel needle assembly with a reverse-bevel technology (EchoTip ProCore) was introduced in 2010-2011. According to the designer, the side notch (not Tru-Cut) should provide additional "cheese-grating" action, making it possible to obtain more specimens.

Initially, a 19G version was introduced to the market. It had the ability to obtain full histology in $89.5 \%$ of cases, with an overall diagnostic accuracy of $93 \%$ [4]. However, because technical difficulties were encountered during transduodenal passes, the same

\section{Video 2}

Endoscopic ultrasound-guided fine-needle aspiration of a pancreatic mass with a 22G EchoTip needle.

online content including video sequences viewable at:

www.thieme-connect.de 


\begin{tabular}{|c|c|c|c|c|}
\hline & $\begin{array}{l}\text { 25G EchoTip ProCore, } \\
\text { median (range) }\end{array}$ & $\begin{array}{l}\text { 22G EchoTip, } \\
\text { median (range) }\end{array}$ & $P$ value & $\begin{array}{l}\text { Table } 1 \text { Histocytologic and } \\
\text { endoscopic assessment of the }\end{array}$ \\
\hline Cytologic score & $1.5(0-3)$ & $2(0-3)$ & $0.438^{1}$ & \\
\hline Histology score & $1(0-2)$ & $1(0-3)$ & $0.220^{1}$ & \\
\hline Total histocytologic score & $2.5(0-5)$ & $3(0-6)$ & $0.255^{2}$ & \\
\hline Macroscopic quantity & $2(1-3)$ & $2(1-3)$ & $0.858^{1}$ & \\
\hline Amount of blood contamination & $1(1-2)$ & $1(1-3)$ & $0.705^{1}$ & \\
\hline Difficulties in visualization & $16 \%$ & $0 \%$ & $0.125^{3}$ & \\
\hline Difficulties in use & $17.3 \%$ & $8.6 \%$ & $0.688^{3}$ & \\
\hline
\end{tabular}

Table 2 Adequacy of material for cytologic and histologic assessment.

\begin{tabular}{|lllllll} 
& \multicolumn{2}{c}{ Adequate material for cytologic assessment } & \multicolumn{4}{c}{ Adequate material for histologic assessment } \\
\hline Target lesion & 25G EchoTip ProCore & 22G EchoTip & P value ${ }^{1}$ & 25G EchoTip ProCore & 22G EchoTip & P value ${ }^{1}$ \\
\hline Pancreatic mass & $16 / 19$ & $15 / 19$ & 1 & $15 / 19$ & $17 / 19$ & 0.68 \\
\hline Lymph node & $8 / 9$ & $7 / 9$ & 1 & $7 / 9$ & $7 / 9$ & 1 \\
\hline All lesions & $24 / 28$ & $22 / 28$ & 0.5 & $22 / 28$ & $24 / 28$ & 0.5
\end{tabular}

${ }^{1}$ McNemar's test.

Table 3 Published comparative trials of the performance of the 22G EchoTip ProCore needle versus the 22G standard EchoTip cytology needle.

\begin{tabular}{|c|c|c|c|c|c|c|c|}
\hline Author & $\begin{array}{l}\text { Type of } \\
\text { study }\end{array}$ & $\begin{array}{l}\text { Number of } \\
\text { lesions }\end{array}$ & Target & $\begin{array}{l}\text { Comparison } \\
\text { of histology } \\
\text { specimen }\end{array}$ & $\begin{array}{l}\text { Overall diagnostic } \\
\text { yield }\end{array}$ & Technical performance & Comment \\
\hline $\begin{array}{l}\text { Bang et al. } \\
2012 \text { [7] }\end{array}$ & Randomized & $\begin{array}{l}28 \text { lesions per } \\
\text { needle type }\end{array}$ & Pancreas & $\begin{array}{l}\text { No significant } \\
\text { difference }\end{array}$ & Equivalent & No significant difference & $\begin{array}{l}\text { Needles of different } \\
\text { manufacturers } \\
\text { (Boston Scientific and } \\
\text { Cook) }\end{array}$ \\
\hline $\begin{array}{l}\text { Witt et al. } \\
2013 \text { [8] }\end{array}$ & $\begin{array}{l}\text { Retrospec- } \\
\text { tive }\end{array}$ & $\begin{array}{l}18 \text { lesions per } \\
\text { needle type }\end{array}$ & $\begin{array}{l}\text { Pancreas, } \\
\text { lymph } \\
\text { nodes, other } \\
\text { masses }\end{array}$ & $\begin{array}{l}\text { No significant } \\
\text { difference }\end{array}$ & $\begin{array}{l}\text { Equivalent; fewer } \\
\text { passes needed with } \\
\text { ProCore needle }\end{array}$ & No reported difficulties & $\begin{array}{l}\text { Retrospective study, } \\
\text { small number per } \\
\text { group }\end{array}$ \\
\hline $\begin{array}{l}\text { Strand et } \\
\text { al. } 2014 \\
{[9]}\end{array}$ & Randomized & $\begin{array}{l}32 \text { lesions } \\
\text { punctured by } \\
\text { both needles }\end{array}$ & Pancreas & $\begin{array}{l}\text { No significant } \\
\text { difference }\end{array}$ & $\begin{array}{l}\text { Lower overall diag- } \\
\text { nostic yield for the } \\
\text { ProCore needle }\end{array}$ & $\begin{array}{l}\text { Technical failure in } 16 \% \\
\text { cases with the ProCore } \\
\text { needle }\end{array}$ & $\begin{array}{l}\text { Only two passes per- } \\
\text { mitted for the } 22 \mathrm{G} \\
\text { ProCore group }\end{array}$ \\
\hline
\end{tabular}

needle was later introduced in a $22 \mathrm{G}$ platform. A PubMed search revealed only three studies of ProCore needles comparing the diagnostic yield of the most commonly used 22G FNA needle with that of the 22G ProCore needle $(-$ Table 3$)$ [7-9]. No advantage of the ProCore design was reported in terms of cytology and histology. However, in the study by Witt et al. [8], fewer passes were needed to achieve diagnosis with the 22G ProCore needle (2.11 with the 22G ProCore needle vs 2.94 with the $22 \mathrm{G}$ standard needle). Nevertheless, this result should be interpreted with caution because of the small number of different groups of lesions ( $\mathrm{n}=$ 18). Interestingly, in the study by Strand et al. [9], technical failure was encountered in 5 of 32 patients because of significant resistance to advancement of the needle, with deflection of the echoendoscope (GF-UCT140, Olympus) while in the transduodenal position.

Most recently, the 25G ProCore needle has been introduced. Potential advantages of the smaller caliber are greater flexibility, less friction in the needle sheath, easier penetration of hard pancreatic tumors, and fewer bloody aspirates. This argument was suggested in a meta-analysis by Madhoun et al. [10] but not confirmed in a recent large randomized controlled trial [14]. Preliminary data on the performance of the $25 \mathrm{G}$ ProCore needle sug- gest an $83 \%$ first-pass sensitivity for pancreatic malignancy, with a cumulative sensitivity of $96 \%$ after three passes [6]. Histologic diagnosis was possible in $63 \%$ after a single pass and in $80 \%$ after multiple passes. In our study, both the $22 \mathrm{G}$ standard needle and the 25G ProCore needle had a slightly better single-pass performance. Cytologic and histologic diagnoses were possible in $84.2 \%$ and $78.9 \%$ of cases, respectively, with an $89.5 \%$ overall sensitivity for pancreatic cancer.

To the best of our knowledge, this is the first randomized, singlegroup, prospective study to assess the diagnostic yield of the $25 \mathrm{G}$ ProCore versus the $22 \mathrm{G}$ standard cytology needle in pancreatic mass lesions and lymphadenopathy adjacent to the upper gastrointestinal tract. Our pilot study compared different needle characteristics and the adequacy of cytology and histology. Although the $25 \mathrm{G}$ needle was not visualized optimally in $16 \%$ of punctures no effect on the quality of cytology or histology, amount of blood contamination, and technical difficulty was observed. We found no significant differences between the two needles in terms of diagnostic yield, with similar diagnostic rates and similar numbers of successful procedures.

Our prospective, single-group, paired design has the intrinsic strength that performance comparisons were made on the same 

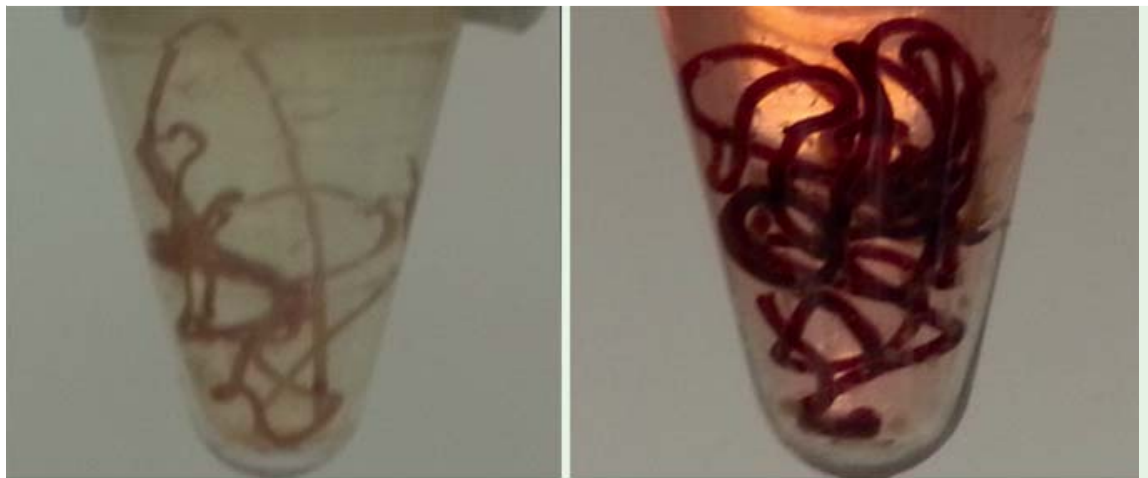

Fig. 2 Macroscopic (top), cytologic (center), and histologic (bottom) images of endoscopic ultrasound-guided fine-needle aspiration specimens of pancreatic adenocarcinoma punctured with a 25G EchoTip ProCore (left) and a 22G EchoTip cytology needle (right).

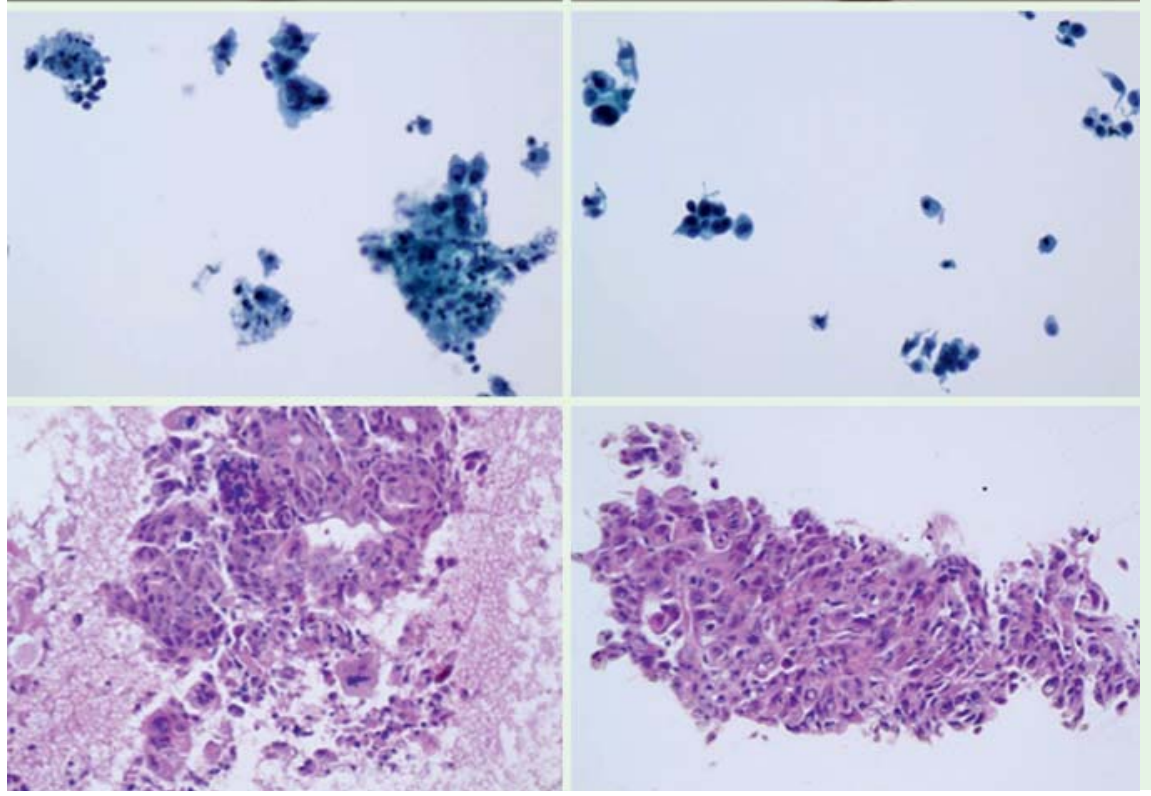

\section{Acknowledgment}

Dr. Mavrogenis wishes to express his gratitude to his EUS teachers (Drs. Warzée, Sibille, Hassaini, and Deprez) and to his wife for her patience and support.

\section{References}

1 Wiersema MJ, Vilmann P, Giovannini $M$ et al. Endosonography-guided fine-needle aspiration biopsy: diagnostic accuracy and complication assessment. Gastroenterology 1997; 112: 1087-1095

2 Gress FG, Hawes RH, Savides TJ et al. Endoscopic ultrasound-guided fine-needle aspiration biopsy using linear array and radial scanning endosonography. Gastrointest Endosc 1997; 45: 243-250

3 Gress F, Gottlieb K, Sherman S et al. Endoscopic ultrasonography-guided fine-needle aspiration biopsy of suspected pancreatic cancer. Ann Intern Med 2001; 134: 459-464

4 Iglesias-Garcia J, Poley JW, Larghi A et al. Feasibility and yield of a new EUS histology needle: results from a multicenter, pooled, cohort study. Gastrointest Endosc 2011; 73: 1189-1196

5 Giovannini M, Monges GM, Iglesias-Garcia J et al. Prospective multicenter evaluation of a novel 22-G Echo-Tip Procore histology EUS-needle in patients with a solid pancreatic mass [abstract 796]. Gastrointest Endosc 2011; 73 : AB152-AB153

6 Iwashita T, Nakai Y, Samarasena JB et al. High single-pass diagnostic yield of a new 25-gauge core biopsy needle for EUS-guided FNA biopsy in solid pancreatic lesions. Gastrointest Endosc 2013; 77: 909-915

7 Bang JY, Hebert-Magee S, Trevino J et al. Randomized trial comparing the 22-gauge aspiration and 22-gauge biopsy needles for EUS-guided sampling of solid pancreatic mass lesions. Gastrointest Endosc 2012; 76: $321-327$ 
8 Witt BL, Adler DG, Hilden K et al. A comparative needle study: EUS-FNA procedures using the HD ProCore ${ }^{\mathrm{TM}}$ and EchoTip ${ }^{\circledR} 22$-gauge needle types. Diagn Cytopathol 2013; 41: 1069-1074

9 Strand DS, Jeffus SK, Sauer BG et al. EUS-guided 22-gauge fine-needle aspiration versus core biopsy needle in the evaluation of solid pancreatic neoplasms. Diagn Cytopathol 2014; 42: 751 - 758

10 Madhoun MF, Wani SB, Rastogi A et al. The diagnostic accuracy of 22gauge and 25-gauge needles in endoscopic ultrasound-guided fine needle aspiration of solid pancreatic lesions: a meta-analysis. Endoscopy 2013; 45: 86-92
11 Imazu $H$, Uchiyama Y, Kakutani $H$ et al. A prospective comparison of EUS-guided FNA using 25-gauge and 22-gauge needles. Gastroenterol Res Pract 2009; 2009: 546390

12 Lee JK, Choi JH, Lee KH et al. A prospective, comparative trial to optimize sampling techniques in EUS-guided FNA of solid pancreatic masses. Gastrointest Endosc 2013; 77: 745 - 751

13 Levy MJ, Wiersema MJ. EUS-guided Trucut biopsy. Gastrointest Endosc 2005; 62: 417-426

14 Vilmann P, Săftoiu A, Hollerbach $S$ et al. Multicenter randomized controlled trial comparing the performance of 22 gauge versus 25 gauge EUS-FNA needles in solid masses. Scand J Gastroenterol 2013; 48: $877-883$ 